\title{
Use of Three-Dimensional Printing to Fabricate First 吅器 a Pelvic Model and Then a Semi-Pelvic Prosthesis 留 with Sacrum: A Case Report
}

\author{
Xin Zhao, Xiaonan Wang, Yu Sun, Meng Xu, Yanbing Wang* and Jincheng Wang* \\ Orthopedic Department, JilinUniversity Second Hospital, China \\ Received: May 06, 2018; Published: May 10, 2018 \\ *Corresponding author: Yanbing Wang, Orthopedic Department, Jilin University Second Hospital, Changchun, PR. China \\ Jincheng Wang, Orthopedic Department, Jilin University Second Hospital, Changchun, PR. China
}

Abbreviations: 3D: Three-Dimensional; CT: Computed Tomography; MRI: Magnetic Resonance Imaging

\section{Introduction}

With continued advancements in medical technology, the focus of treatment develop can shift from making an operation possible to achieving better long-term outcomes, and this means a greater emphasis on the accuracy of medical treatments. Threedimensional (3D) printing is becoming a more mature technology, and its use is becoming more widespread. In the field of orthopedic diagnosis and treatment, 3D printing offers advantages for the accurate preoperative design of a prosthesis, the manufacturing of an individualized prosthesis, and simulating surgery [1-13]. The results of computed tomography (CT), magnetic resonance imaging (MRI), and some other radiographic techniques provide the basis for fabricating structures via 3D printing. Several reports have described the use of 3D printing to model complex fractures of the pelvis and acetabulum $[14,15]$, and other studies $[16,17]$ have used 3D printing to facilitate preoperative planning in patients with developmental dysplasia of the hip. Li et al. used 3D printing to create a customized implant in a case of revision total hip arthroplasty [9]. Here, we present a novel case in which we used 3D printing technology in the preoperative planning of semi-pelvic prosthesis replacement [18]. In this case, we printed a pelvic model based on CT scans and used the model for the preoperative design and simulation of the operation. In addition, the new titanium alloy (Ti-6Al-4V) semi-pelvic prosthesis with sacrum was designed and fabricated via 3D printing.

\section{Case report}

The 51-year-old male patient had previously undergone left semi-pelvic resection of chondrosarcoma and semi-pelvic prosthesis implantation. The patient reported experiencing localized pain in the left hip for 1 year prior to presentation. Ultrasonography revealed an inhomogeneous low-echo mass in the left hip. MRI revealed equal T1 and long T2 signals in the left pelvis. A pelvic radiograph showed loosening of the prosthesis and a broken screw (Figure 1). Aspiration biopsy was conducted and indicated the recurrence of chondrosarcoma. Based on the patients clinical history and biopsy result, the patient was diagnosed with recurrent chondrosarcoma in the left hip. Pelvic CT scanning was performed, and the CT data were imported into the medical image processing software OsirixMD, which was used to build a virtual 3D model of the patient s pelvis. On the virtual model, the loosened prosthesis, broken screw, and recurrent tumor foci could be observed (Figure 2). The CT data were then imported into the medical image processing software MIMICS, and a 3D printed physical model of the pelvis was fabricated using a three-step process: segmentation of the relevant anatomy, development of a virtual model from the segmented anatomy, and physical printing and post-processing of the model (Figure 3). In MIMICS, the bone and prosthesis could be isolated from the other tissue using a threshold filter tool based on the grey level.

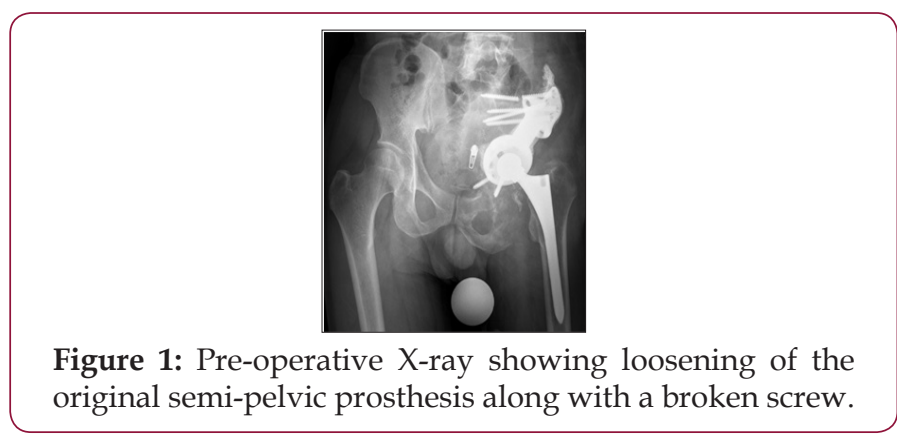




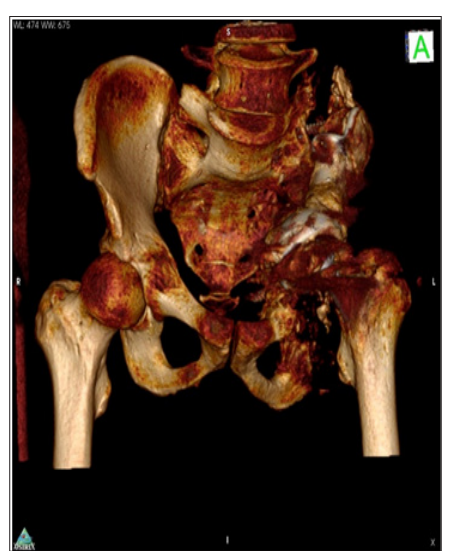

Figure 2: Pre-operative $X$-ray showing loosening of the original semi-pelvic prosthesis along with a broken screw.

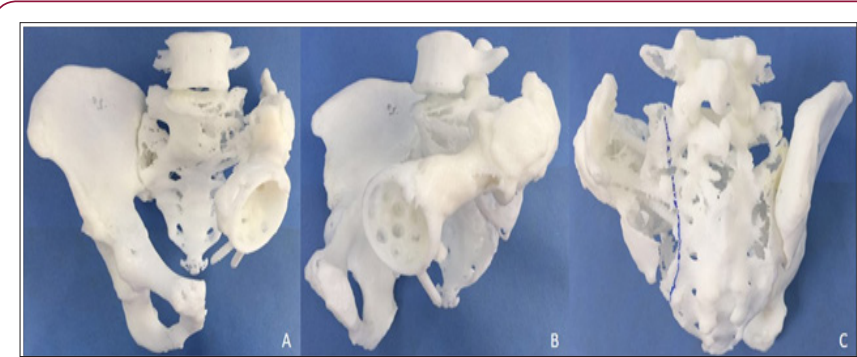

Figure 3: 3D printed physical pelvic model. A: Anterior, B: lateral, and C: posterior views.

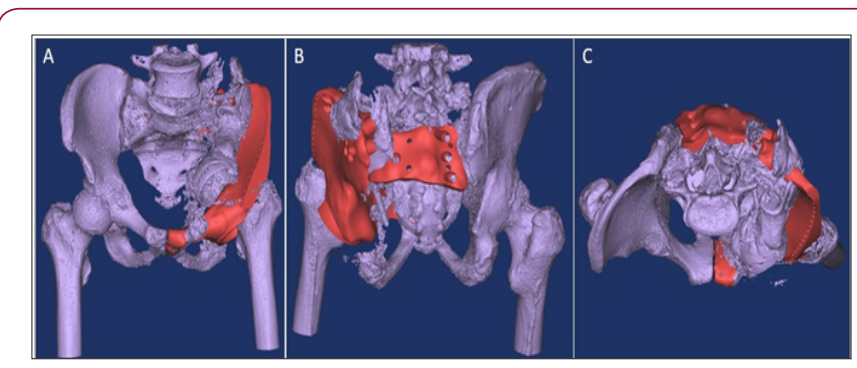

Figure 4: Virtual design of the pelvic prosthesis. A: Anterior, B: posterior, and C: top views.

To ensure the surface continuity of the physical model, regions where partial volumes fell below the threshold had to be segmented manually. The virtual model was exported as an STL file and imported into Net fabb software for 3D printing of the physical model with a photosensitive resin on a SLA 3D printer.The physical pelvic model provided significant direction in pre-operative design of the complex semi-pelvic prosthesis replacement and the fabrication of an individualized prosthesis. Before operation, we were able to assess the excision range of the tumor on the model. We planned to cut off the left sacroiliac joint, pubic symphysis, and left ischial ramus. Then we designed the individualized 3D printed prosthesis according to the excision range and to exactly match the remnant pelvis (Figure 4). In addition, two screw holes were designed on the left and right sacroiliac joint fitting points. These screw hole locations were chosen to ensure rigid fixation between the prosthesis and bone and to provide good initial stability. The 3D printed pelvic prosthesis was fabricated from the titanium alloy
Ti-6Al-4V (Insert information for the supplier of the Ti-6Al-4V here).The operation was performed according to the pre-operative planning. The original pelvic prosthesis and broken screw were removed. The tumor was excised by cutting off the left sacroiliac joint, pubic symphysis, and left ischial ramus. The 3D printed pelvis prosthesis was implanted and secured by two cancellous bone screws on the right and left sacroiliac joint fitting points.

The acetabular component was set up on the pelvic prosthesis, and the hip joint was reset. After completion of the implantation, the hip joint showed a good range of motion and stability. Postoperative X-ray imaging revealed good positioning of the new prosthesis (Figure 5). The patient completed his recovery in an inpatient rehabilitation facility and was discharged home. At 6 months after the operation, the patient had good hip joint function and was able to care for himself. Follow-up CT scanning was performed, and an additional virtual 3D model of the pelvis and prosthesis was produced using OsirixMD (Figure 6). Good prosthesis positioning was confirmed by the model.

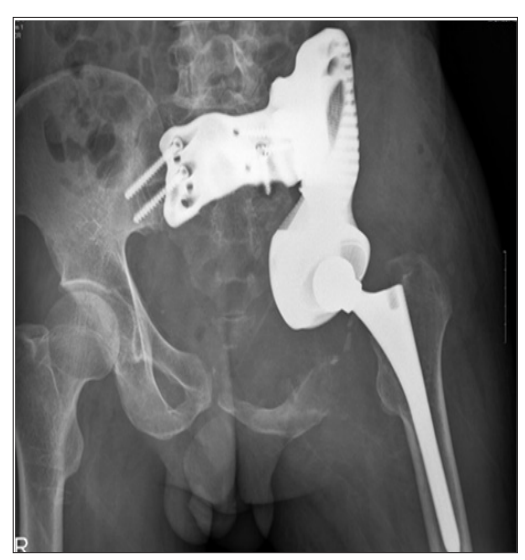

Figure 5: Post-operative $X$-ray image.

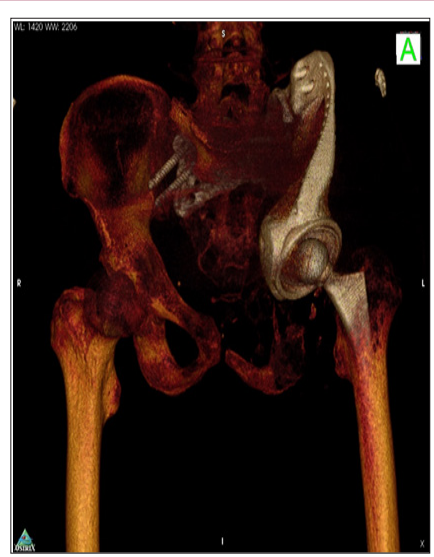

Figure 6: Virtual model of pelvis reconstruction at 6 months after implantation of the 3D printed prosthesis.

\section{Discussion}

This case demonstrates that pre-operative fabrication of a pelvic model using 3D printing based on CT data can help surgeons in the planning of a complex hemi-pelvic replacement. In our patient, the 
use of a such a model in pre-operative planning was significant for maintaining continuity of the pelvis, improving bone ingrowth, and enhancing the stability of the prosthesis. Previously, when surgeons performed semi-pelvic prosthesis replacement, they often had difficulty in determining the appropriate size of the prosthesis and the necessary range of excision. These challenges increased operating times and reduced the likelihood of a satisfactory outcome. The 3D printed model provides opportunities to rehearse the operation and determine more accurately and precisely the necessary range of bone excision. In addition, the screw hole locations can be selected during the simulated operation to provide greater initial stability. The precision of this surgery simulation is established by the accuracy of CT data and depends also on the proficiency of the operating staff.

Furthermore, the 3D printed prosthesis is designed based on the determined range of bone excision, and thus, the shape of the prosthesis can perfectly match the defect left after resection, which serves to maintain continuity of the pelvis.

With the development of 3D printing technology, improvements in the precision of CT scanning, and reductions in the costs of the required materials, the widespread use of $3 \mathrm{D}$ printing to fabricate physical models of bones as well as orthopedic prostheses becomes possible. Currently, the cost of printing and the required manpower are two factors that can limit the use of this technology. At present, the cost for a 3D model of the hip and hemi-pelvis varies depending upon factors such as the materials used, the scale (size) of the print, and the type of printer employed. However, these costs may be offset by the savings in operating-theater time. In the foreseeable future, we expect that the use of 3D printing technology to fabricate orthopedic models based on CT data and then to manufacture prostheses based on the model-guided pre-operative planning could lead to improved workflows, decreased operating times, and better patient outcomes.

\section{Acknowledgement}

The authors are grateful for the financial support from Jilin University (project450060491517) and Ministry of Education of the People's Republic of China (project3M213BV43430).

\section{References}

1. Bizzotto N, Sandri A, Regis D, Romani D, Tami I, et al. (2015) ThreeDimensional Printing of Bone Fractures: A New Tangible Realistic Way for Preoperative Planning and Education. Surgical Innovation 22(5): 548-551.

2. Chung KJ, Hong DY, Kim YT, Yang I, Park YW, et al. (2014) Preshaping Plates for Minimally Invasive Fixation of Calcaneal Fractures Using a Real-Size 3D-Printed Model as a Preoperative and Intraoperative Tool. Foot \& Ankle International 35(11): 1231-1236.

3. Ciocca L, De Crescenzio F, Fantini M, Scotti R (2009) CAD/CAM and rapid prototyped scaffold construction for bone regenerative medicine and surgical transfer of virtual planning: a pilot study. Computerized Medical Imaging \& Graphics the Official Journal of the Computerized Medical Imaging Society. 33(1): 58-62.

4. Giovinco NA, Dunn SP, Dowling L, Smith C, Trowell L, et al. (2012) A Novel Combination of Printed 3-Dimensional Anatomic Templates and Computer-assisted Surgical Simulation for Virtual Preoperative Planning in Charcot Foot Reconstruction. Journal of Foot \& Ankle Surgery Official Publication of the American College of Foot \& Ankle Surgeons 51(3): 387-393.

5. Gittard SD, Narayan RJ, Lusk J, Morel P, Stockmans F, et al. (2009) Rapid prototyping of scaphoid and lunate bones. Biotechnology Journal 4(1): 129-134.

6. Guarino J, Tennyson S, McCain G, Bond L, Shea K, et al. (2007) Rapid prototyping technology for surgeries of the pediatric spine and pelvis: benefits analysis. Journal of Pediatric Orthopedics 27(8): 955-960.

7. Holubar SD, Hassinger JP, Dozois EJ, Camp JC, Farley DR,et al. (2009) Virtual pelvic anatomy and surgery simulator: an innovative tool for teaching pelvic surgical anatomy. Studies in Health Technology \& Informatics 142: 122-124.

8. Jastifer JR, Gustafson PA (2017) Three-Dimensional Printing and Surgical Simulation for Preoperative Planning of Deformity Correction in Foot and Ankle Surgery. Journal of Foot \& Ankle Surgery Official Publication of the American College of Foot \& Ankle Surgeons 56(1): 191-195.

9. Li H, Qu X, Mao Y, Dai K, Zhu Z (2016) Custom Acetabular Cages Offer Stable Fixation and Improved Hip Scores for Revision THA With Severe Bone Defects. Clinical Orthopaedics \& Related Research 474(3): 731740 .

10. Ma L, Zhou Y, Zhu Y, Lin Z, Wang Y, et al. (2016) 3D-printed guiding templates for improved osteosarcoma resection. Scientific Reports 6: 23335.

11. McGurk M, Amis AA, Potamianos P, Goodger NM (1997) Rapid prototyping techniques for anatomical modelling in medicine. Annals of the Royal College of Surgeons of England 79(3): 169-174.

12. Windisch G, et al. (2007) A model for clubfoot based on micro-CT data. Journal of Anatomy 210(6): 761-766.

13. Jahnke P, Limberg FR, Gerbl A, Ardila Pardo GL, Braun VP, et al. (2017) Radiopaque Three-dimensional Printing: A Method to Create Realistic CT Phantoms. Radiology 282(2): 569-575.

14. Zeng C, Xiao J, Wu Z, Huang W (2015) Evaluation of three-dimensional printing for internal fixation of unstable pelvic fracture from minimal invasive para-rectus abdominis approach: a preliminary report. International Journal of Clinical \& Experimental Medicine 8(8): 1303913044.

15. Hurson C, Tansey A, O’Donnchadha B, Nicholson P, Rice J, et al. (2007) Rapid prototyping in the assessment, classification and preoperative planning of acetabular fractures. Injury-international Journal of the Care of the Injured 38(10): 1158-1162.

16. Yang J, Zhang LN, Fei LI, Wang CW, Liu DP (2015) Application of digital navigation system in treatment of adult DDH surgery. Chinese Journal of Bone \& Joint Injury.

17. Yan H, Shi Q WU Wei-Ping (2015) Application of 3D Printing Technology in the Revision Surgery for DDH in Children. China Digital Medicine.

18. Xu J, Li D, Ma RF, Barden B, Ding Y (2015) Application of Rapid Prototyping Pelvic Model for Patients with DDH to Facilitate Arthroplasty Planning: A Pilot Study. Journal of Arthroplasty 30(11): 1963-1970. 


\section{(C) (i) This work is licensed under Creative}

Submission Link: https://biomedres.us/submit-manuscript.php

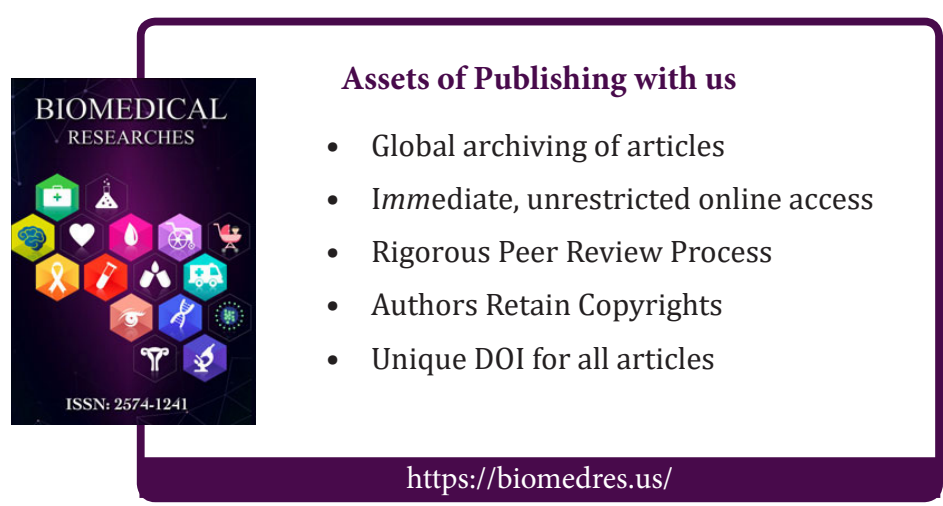

